\title{
Lactate UV-system: a rapid method for diagnosis of septic arthritis
}

\author{
A. R. BEHN, J. A. MATHEWS, AND I. PHILliPS \\ From the Departments of Rheumatology and Microbiology, St Thomas's Hospital, London SE1 7EH
}

SUMMARY The concentration of lactic acid in synovial fluid was estimated in 43 specimens from patients with an acute monoarthritis by a simple enzyme method. In 9 patients with 10 episodes of septic arthritis concentrations of synovial fluid lactic acid were significantly higher (mean 10.8 $\mathrm{mmol} / \mathrm{l}$ ) than in 33 patients with nonseptic effusions (mean $3 \cdot 1 \mathrm{mmol} / \mathrm{l}$ ). With this method concentrations of synovial fluid lactic acid provide a rapid diagnostic guide in the separation of septic from nonseptic arthritis.

Septic arthritis causes rapid destruction of a joint if not recognised and treated promptly. Patients with an acute monoarticular arthritis, even when there is a history of a chronic polyarthritis, should be suspected of having joint infection and provide a diagnostic problem. Synovial fluid contains pus cells in all inflammatory arthropathies, and an immediate Gram stain detects the bacterial cause in only a proportion of infected cases. ${ }^{1}$ Synovial fluid culture results are usually available after a delay of 24 to 48 hours and occasionally fail to yield a diagnosis. This is a particular problem when the infecting organism has fastidious growth requirements, for example, Neisseria gonorrhoeae or Haemophilus influenzae, ${ }^{2}$ and when patients have been partially treated with antiboitics. ${ }^{3}$

Qualitative $^{4}$ and quantitative ${ }^{5}$ analysis has shown raised levels of synovial fluid lactic acid in septic arthritis, suggesting an additional technique for detecting acute bacterial infection. Previous studies have used gas-liquid chromatography to assess lactic acid levels. We have used an enzymatic method to estimate synovial fluid lactic acid concentrations in cases of acute monoarticular arthritis.

\section{Patients and methods}

Forty-three episodes of acute monoarthritis were studied in 42 patients. All were examined clinically, radiologically, and by laboratory investigation to establish a diagnosis. Eighteen of the patients were female and 24 were male. Their ages ranged from 21 to 76 years (mean 49 years). Specimens of synovial

Accepted for publication 15 December 1980. Correspondence to Dr A. R. Behn. fluid were collected aseptically from 32 knees, 3 shoulders, 3 ankles, 2 elbows, 2 wrists, and 1 hip. The specimens were examined by microscopy and Gram stain. Routine culture was carried out on blood agar under aerobic and anaerobic conditions, and on lysed blood agar with added vancomycin, colistin, nystatin, and trimethoprim for $N$. gonorrhoeae. Total synovial fluid white cell counts were estimated on fresh sequestrene specimens with a Coulter counter. An aliquot of fluid was frozen at $-20^{\circ} \mathrm{C}$ in a sterile container until analysed for lactic acid. Other techniques such as polarised light microscopy were used when necessary.

\section{LACTATE $+\mathrm{NAD}^{+} \stackrel{\text { LDH }}{\rightleftharpoons}$ PYRUVATE $+\mathrm{NADH}^{+}$ $+\mathrm{H}^{+}$ \\ PYRUVATE + GLUTAMATE GPT ALANINE + OXOGLUTARATE}

Fig. 1 Chemical principle of the lactate UV-system.

The lactate UV-system (Boehringer-Mannheim) was used to measure synovial fluid lactic acid concentration. This recently developed method of assay ${ }^{6}$ is based on the oxidation of lactate, catalysed by lactate dehydrogenase (LDH) to pyruvate (Fig. 1). During this oxidation equimolar concentrations of reduced nicotinamide adenine dinucleotide (NADH) are produced and can be quantitated in an ultraviolet spectrophotometer. To ensure that this reaction is complete, pyruvate is converted by glutametapyruvate transaminase (GPT) to alanine.

A Pye-Unicam SP8-100 ultraviolet spectrophotometer was used at a wavelength of $340 \mathrm{~nm}$ to measure absorbance of each sample and its sample 
blank. Specimens did not need deproteinisation. Approximately 20 minutes was required to complete the assay.

Quality control assessment with a standard (Precinorm S, Boehringer-Mannheim), whose actual lactate concentration was $3.26 \mathrm{mmol} / \mathrm{l}$, gave a mean value of $3.05 \mathrm{mmol} / \mathrm{l}(\mathrm{n}=20)$, with a coefficient of variation of $8 \%$.

There were 10 episodes of septic arthritis in 9 patients. Eight were attributed to Staphylococcus aureus and 2 to Neisseria gonorrhoeae. A diagnosis of septic arthritis was confirmed by positive synovial fluid culture in 8 out of 10 samples. The diagnosis in 1 gonococcal case in which the synovial fluid was frankly purulent was made on the basis of the isolation of $N$. gonorrhoeae from the cervix and full response to antibiotic treatment alone. In 1 staphylococcal case the organism was cultured from a paronychia 2 days before joint aspiration; the patient had been treated for those 2 days with oral erythromycin and the joint fluid was sterile. This patient's acute monoarthritis responded to antibiotics alone. The mean duration of symptoms in 9 episodes of joint infection was 3.3 weeks (range 2 days to 16 weeks). Two patients had been treated with oral antibiotics for 2 days and 1 day respectively prior to their first joint aspiration. Five patients had a chronic intercurrent illness (chronic renal failure, renal transplantation, systemic sclerosis, rheumatoid arthritis, diabetes mellitus).

Thirty-three patients had a nonseptic monoarthritis. Seven patients had rheumatoid arthritis, 6 a crystal synovitis, 6 Reiter's disease, and 14 an assortment of arthropathies (osteoarthritis, psoriatic, colitic, Behçet's, ankylosing spondylitis, Wegener's granulomatosis). The mean duration of symptoms in 29 was 3.2 weeks (range 2 days to 20 weeks). At presentation 15 patients were being treated with nonsteroidal anti-inflammatory drugs, 3 with anticoagulants, 2 with corticosteroids, 2 with gold injections, and 1 with allopurinol.

\section{Results}

Synovial fluid lactic acid concentrations from patients with septic arthritis were significantly higher than those from patients with nonseptic arthritis (Table 1). Fig. 2 shows the distribution of individual lactate levels. Despite the clear separation of the 2 main groups there are several intermediate values. The lowest level in septic arthritis $(5.7 \mathrm{mmol} / \mathrm{l})$ occurred in the only patient in whom 2 separate episodes were studied. In the first episode before any treatment the knee fluid lactic acid level was $10 \cdot 6$ $\mathrm{mmol} / \mathrm{l}$. One week after stopping flucloxacillin a
Table 1 Synovial fluid lactic acid concentration in 42 patients with an acute monoarticular arthritis

\begin{tabular}{lllc}
\hline Diagnosis & Number of samples & \multicolumn{2}{l}{ Lactic acid mmol/l } \\
\cline { 2 - 4 } & & Range & Mean \\
\hline Septic & 10 & $5 \cdot 7-15 \cdot 2$ & $10 \cdot 8^{*}$ \\
Nonseptic & 33 & $0 \cdot 7-7 \cdot 8$ & $3 \cdot 1^{*}$ \\
\hline
\end{tabular}

${ }^{* \mathrm{p}}<0.001(t=10 \cdot 66)$.

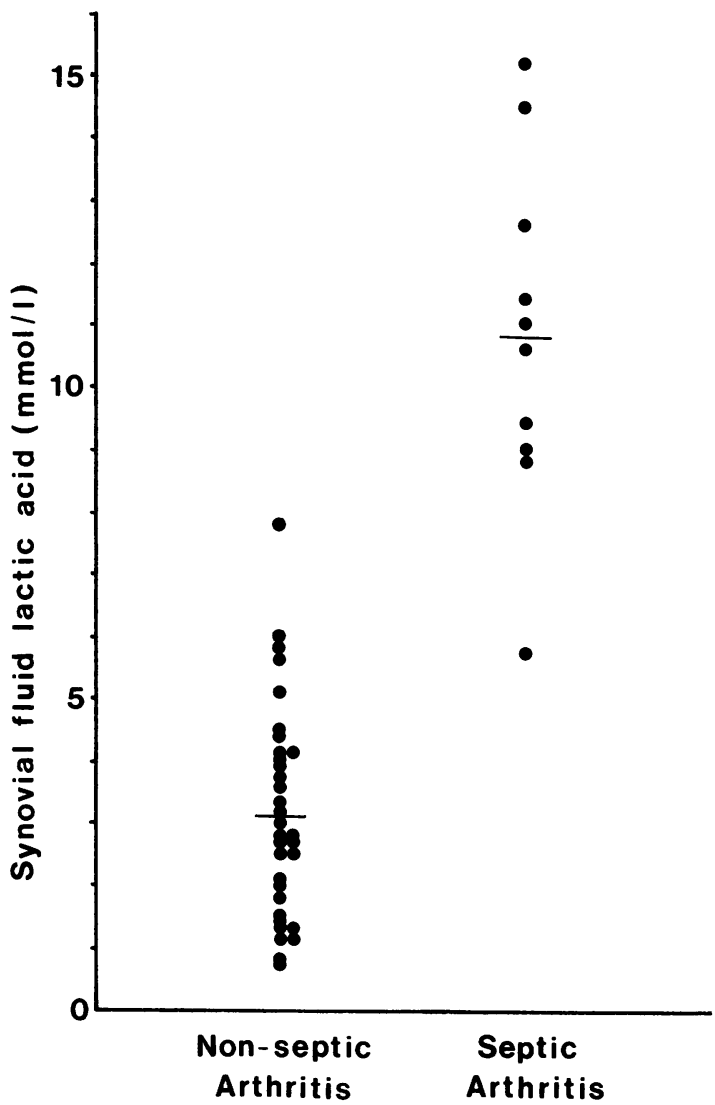

Fig. 2 Synovial fluid lactic acid concentration in 10 episodes of septic arthritis (staphylococcal and gonococcal) and 33 episodes of nonseptic arthritis. (Conversion of SI to traditional units: Lactic acid concentration $1 \mathrm{mmol} / \mathrm{l}=9 \mathrm{mg} / 100 \mathrm{ml}$ ).

further episode of septic arthritis occurred in an ankle, the synovial fluid lactic acid was $5.7 \mathrm{mmol} / \mathrm{l}$, and the subsequent culture report was of a scanty growth of staphylococcus aureus. Lactic acid levels were raised in synovial fluid from both the cases negative on gonococcal culture $(9.4 \mathrm{mmol} / \mathrm{l})$ and positive $(11.0 \mathrm{mmol} / \mathrm{l})$. 
There were 2 cases of septic arthritis in which conventional culture techniques were negative at the time of presentation. In the first of these the presumptive bacteriological diagnosis was made by culture of Staph. aureus from a paronychia and the synovial fluid lactic acid level was elevated to $14.5 \mathrm{mmol} / \mathrm{l}$. In the second a subsequent synovial fluid culture grew Staph. aureus, and the initial synovial fluid lactic acid level was already elevated to $8.8 \mathrm{mmol} / \mathrm{l}$. In both cases the subsequent clinical course was characteristic of septic arthritis.

In 2 cases of staphylococcal infection in which the joint was serially aspirated before and during treatment lactic acid levels decreased (Fig. 3), in one (case 1) after combined intra-articular flucloxacillin, intravenous flucloxacillin, and fusidic acid, in the other (case 2) after intravenous flucloxacillin and fusidic acid.

The mean total white cell count in synovial fluid from 16 nonseptic cases was $7 \cdot 7 \times 10^{9} / 1$ (range

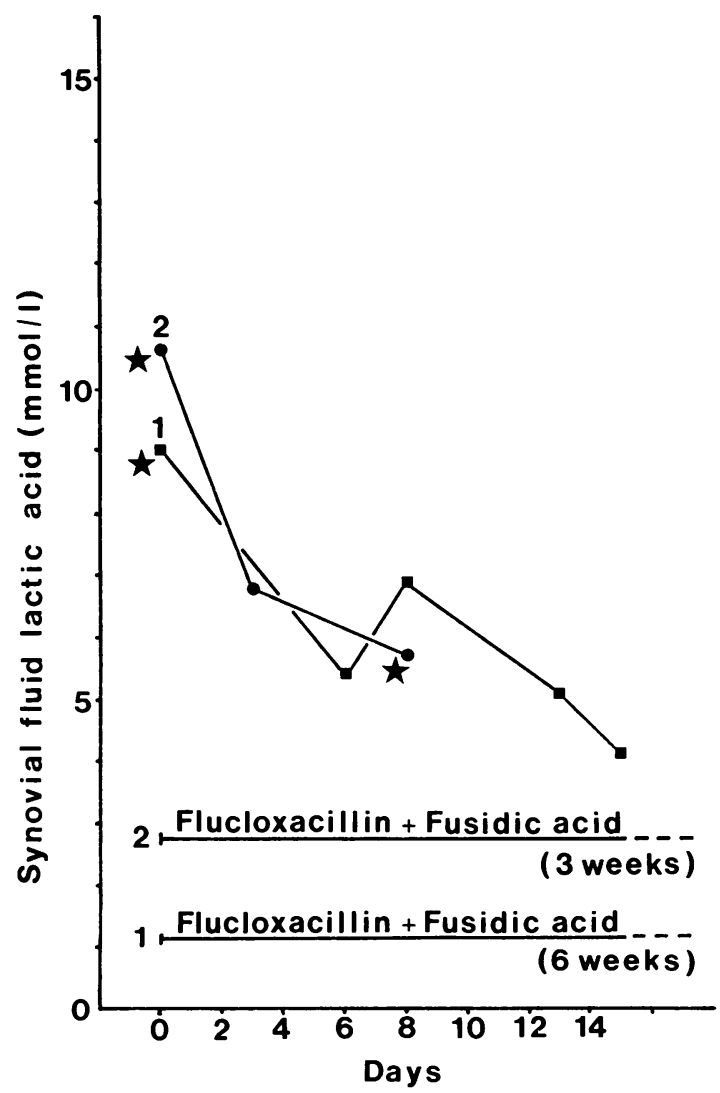

Fig. 3 Change in synovial fuid lactic acid concentration with treatment in 2 cases of staphylococcal arthritis. $\star$ Indicates culture positive synovial fluid. $\left.0 \cdot 6-15 \cdot 4 \times 10^{9} / 1\right)$ and from 4 septic cases was $53.9 \times 10^{9} / 1$ (range 31.0-75.9 $\times 10^{9} / 1$ ). A significant positive correlation was found between the total synovial fluid white cell count and the synovial fluid lactic acid $(\mathrm{r}=0 \cdot 83, t=6 \cdot 49, \mathrm{p}<0 \cdot 001)$.

\section{Discussion}

Brooks et al. ${ }^{7}$ have analysed synovial fluids, which had been extracted and derivatised, using gas-liquid chromatography. Complex elution patterns were produced which were sufficiently dissimilar to permit differentiation between traumatic and septic arthritis. Seifert et al. ${ }^{4}$ used the simpler and quicker technique of direct analysis of synovial fluid by gas-liquid chromatography without extraction or derivatisation. They showed qualitatively the presence of lactic acid in excess of a concentration of $10 \mathrm{mmol} / \mathrm{l}$ in 5 culture-positive joint fluids and in 2 culture-negative cases of gonococcal arthritis.

Our results with the lactate UV-system also show significantly higher levels of lactic acid in septic joint fluid, including those from culture-positive and negative gonococcal arthritis. It appears likely that partial or inadequate antibiotic treatment will lower lactate levels, as in 1 staphylococcal case in which 2 episodes were studied. In the 2 cases from which serial synovial fluid samples were taken before and during treatment the lactic acid concentration fell within 6 days.

Brook et al. ${ }^{5}$ used gas-liquid chromatography to quantitate synovial fluid lactic acid in 84 patients with an acute monoarthritis. They found high levels in 27 cases of nongonococcal septic arthritis (range 5.3-277 $\mathrm{mmol} / \mathrm{l}$ ) and low levels in 45 cases of inflammatory or degenerative arthritis (range $1 \cdot 0-6 \cdot 9 \mathrm{mmol} / \mathrm{l})$. However, low levels $(2 \cdot 0-5 \cdot 6$ $\mathrm{mmol} / \mathrm{l}$ ) were found in synovial fluid from 12 patients with gonococcal arthritis, of whom 8 had positive synovial fluid cultures. The disadvantages of gas-liquid chromatography are the tediousness of extraction and derivatisation of specimens for quantitative analysis.

Studies of metabolism in rheumatoid synovial fluid $^{8}$ have shown that reduction in partial pressure of oxygen $\left(\mathrm{Po}_{2}\right)$ is accompanied by reduction of $\mathrm{pH}$ and elevation in partial pressure of carbon dioxide $\left(\mathrm{PCO}_{2}\right)$.

Treuhaft and $\mathrm{McCarty}{ }^{9}$ have shown that a fall in synovial fluid $\mathrm{Po}_{2}$ and $\mathrm{pH}$ level correlates with a rise in lactic acid concentration in a group of 18 patients, 12 of whom had inflammatory joint disease. They suggest this is the result of a change from aerobic to anaerobic glycolysis by a hypoxic synovium. In their study the mean synovial fluid lactic acid in 12 
patients with nonseptic inflammatory arthritis was $3.3 \mathrm{mmol} / \mathrm{l}$ (range $1 \cdot 7-6.2 \mathrm{mmol} / \mathrm{l}$ ).

The mechanism by which joint infection leads to higher concentrations of lactic acid in synovial fluid is not entirely clear. We found a significant positive correlation between the total synovial fluid white cell count and lactic acid concentrations in 20 samples. Treuhaft and McCarty's results ${ }^{9}$ on 17 samples of synovial fluid from noninfected joints show a weak correlation between white cell count and lactic acid concentration. This might suggest that synovial tissue hypoxia and lactic acid concentration are related to the severity of the inflammatory process as reflected by the synovial fluid white cell count. However, in the same study the negative correlation between $\mathrm{Po}_{2}$ and synovial fluid white cell count was poor. Brook et al. ${ }^{5}$ showed overlap in the synovial fluid white cell count between patients with nonseptic inflammatory, gonococcal, and nongonococcal septic arthritis and yet significant increase of lactic acid levels in the nongonococcal septic group.

There is further evidence that the presence of bacteria exerts an additional influence from in-vitro experiments studying the effects of bacterial products on selected synovial fibroblast culture. ${ }^{10}$ The addition of Gram-negative bacterial extracts increased glucose utilisation and lactic acid production. It is unlikely that the white cells themselves are the source of the lactic acid. In-vitro studies have shown that as many as $10^{6}$ endotoxin-stimulated polymorphonuclear leucocytes yield only small quantities of lactic acid.11

We conclude that the lactate UV-system is a quick and simple method of assay for measuring synovial fluid lactic acid. We have used it to confirm that levels are elevated in the presence of bacterial infection.
This project was supported by a grant from the Arthritis and Rheumatism Council.

\section{References}

1 Goldenberg D L, Cohen A S. Acute infectious arthritis. A review of patients with non-gonococcal joint infections. Am J Med 1976; 60: 369-77.

"Sommers H M. The microbiology laboratory in the diagnosis of infectious arthritis. Clin Rheum Dis 1978; 4: 63-82.

3 Newman J H. Review of septic arthritis through the antibiotic era. Ann Rheum Dis 1976; 35: 198-205.

4 Seifert M H, Mathews J A, Phillips I, Gargan R A. Gas liquid chromatography in diagnosis of pyogenic arthritis. Br Med J 1978; ii: 1403.

5 Brook I, Reza M J, Bricknell K S, Bluestone R, Finegold S M. Synovial fluid lactic acid. Alagnostic aid in septic arthritis. Arthritis Rhcum 1978; 21 : 774-9.

6 Noll $F$ L-!actate. determination with LDH, GPT and NAD. In: Bergmeyer H U, ed. Methods of Enzymatic Analysis. 2nd ed. New York and London: Chemie Weinheim and Academic Press, 1974: 1475-7.

7 Brooks J D, Kellogg D S, Alley C C, Short H B, Handsfield $\mathrm{H} \mathrm{H}$, Baker H. Gas chromatography as a potential means of diagnosing arthritis. Differentiation between staphylococcal, streptococcal, gonococcal and traumatic arthritis. $J$ Infect Dis 1974; 129 : 660-8.

8 Lund-Olsen $K$. Oxygen tension in synovial fluids. Arthritis Rheum 1970; 13: 769-76.

9 Treuhaft P S, McCarty D J. Synovial fluid pH, lactate, oxygen and carbon dioxide partial pressure in various joint diseases. Arthritis Rheum 1971 ; 14: 475-84.

10 Buckingham R B, Caster C W, Hoag P F. The effect of bacterial products on synovial fibroblast function: hypermetabolic changes induced by endotoxin. $J$ Clin Invest $1972 ; 51$ : 1186-94.

11 Cohn Z A, Morse S I. Functional and metabolic properties of polymorphonuclear leucocytes. II. The influence of a lipopolysaccharide endotoxin. J Exp Med 1960; 111: 689-704. 